\title{
A SIMPLE MODEL FOR RANDOM OSCILLATIONS
}

\author{
F. PAPANGELOU, ${ }^{*}$ University of Manchester
}

\begin{abstract}
A simple model for a randomly oscillating variable is suggested, which is a variant of the two-state random velocity model. As in the latter model, the variable keeps rising or falling with constant velocity for some time before randomly reversing its direction. In contrast however, its propensity to reverse depends on its current value and it is for this desirable feature that the model is proposed here. This feature has two implications: (a) neither the changing variable nor its velocity is Markovian, although the joint process is, and (b) the linear differential equations arising in the case of our model do not have constant coefficients. The results given in this paper are meant to illustrate the straightforward nature of some of the calculations involved and to highlight the relationship with onedimensional diffusions.

Keywords: Random oscillation; random evolution; inertia; hazard field; Markov process; generator; stationary distribution; renewal; level crossing; recurrence time; exit distribution; exit time; diffusion; scale and speed measure
\end{abstract}

2010 Mathematics Subject Classification: Primary 60K40

Secondary $60 \mathrm{~J} 25$

\section{Introduction}

The purpose of the present paper is to suggest a model for random oscillations in one dimension which is simple, reasonably discrete, amenable to calculation, and not entirely unrealistic. There are many systems that undergo random expansion and contraction, and whose swings are reflected in a numerical index which rises or falls as the system grows or retrenches. Our object of study will be such an index, which we will imagine as a 'particle' moving up or down the real line in a manner that incorporates the two principles mentioned in the abstract above, which are explained more precisely below.

Our model is inspired by the two-state random velocity model, which is the most basic 'random evolution' and is lucidly presented in Chapter 0 of [4]. In that model a particle moves on the line with constant velocity, either $\gamma$ or $-\gamma$ say, switching from one velocity to the other at random times given by a Poisson process of rate $\lambda$. It is more suggestive to imagine the velocity process as a two-state Markov chain which 'instructs' the particle to adopt one or the other velocity and proceeds regardless of where the particle is at any given moment. To describe the motion informally, we can say that at any given time $t$ the particle, which is moving with constant speed, has probability $\lambda \Delta t+o(\Delta t)$ of reversing its direction of motion before time $t+\Delta t(\Delta t \rightarrow 0)$. The position process of the particle is not Markovian, but the velocity process as well as the position-cum-velocity process are. This idea has been greatly generalised to that of random evolution, where an (often discrete) Markovian environment drives the development of a system without any feedback from the 'position' of the system. See [4] and the references given therein for the theory and many applications of such processes.

Received 15 February 2010; revision received 12 September 2010.

* Postal address: School of Mathematics, University of Manchester, Manchester, M13 9PL, UK.

Email address: fredos.papangelou@manchester.ac.uk 
The model studied here is a two-state velocity model, but with a modification which is an essential ingredient and a defining characteristic of the process. The modification is this: if at time $t$ the particle is at position $x$ and is moving up, then it has probability $r(x) \Delta t+o(\Delta t)$ of reversing its motion before time $t+\Delta t$, whereas if it is moving down it has probability $l(x) \Delta t+o(\Delta t)$ of reversing before time $t+\Delta t$. Here $r(x)$ and $l(x)$ are functions of the position $x$ of the particle and represent the propensity of the particle to reverse its motion while passing through $x$. If, for any time $t \geq 0$, we denote by $X(t)$ the position and by $V(t)$ the velocity of the particle, then the stochastic process $(X(t), V(t))$ is Markovian, but neither the marginal process $X(t)$ nor $V(t)$ is Markovian. Despite this, the obvious generalisations of the infinitesimal generator of $(X(t), V(t)), t \geq 0$, and the differential equations appearing in [4] hold in this modified context and the interesting thing is that, although these linear differential equations (unlike the case of [4]) do not have constant coefficients, they admit elementary explicit solutions when applied to some of the usual entities of interest in probability, such as stationary distributions, exit distributions, mean exit times, etc.

The crucial aspect of the model is the dependence of the propensity functions $r(\cdot)$ and $l(\cdot)$ on location. To take a simple example, assume that there is a pole at the origin 0 , such that the further away a particle is from 0 the greater its propensity to reverse if it is actually moving away from 0 and the less if it is moving towards 0 . This can be modelled by choosing $r(\cdot)$ to be increasing and $l(x)$ to be equal to $r(-x)$ on $(-\infty, \infty)$. There is an infinity of variations on this theme. In general, we can imagine that, as the particle moves, it senses a 'field' which manifests itself not as a field of forces but as a 'hazard field' defined by the two propensity functions. Thus, the model embodies two features: (a) the particle, whether moving up or down, has a tendency to carry on in the same direction for a while before it reverses ('inertia') and (b) its propensity to reverse depends on location, so that, for instance, a reversal becomes more and more likely as the particle reaches excessively high or low levels, as so often happens with indices in the economic sphere.

This paper is essentially an exploration of the role played by the propensity functions $r(\cdot)$ and $l(\cdot)$, and concentrates on problems which admit explicit closed-form solutions, some of which can be compared with the corresponding solutions for one-dimensional diffusions. It is written partly as a piece of advocacy for the use of state-dependent propensities as modelling tools. After setting up the model and displaying its infinitesimal generator in Section 2, we calculate the stationary distribution of the process $(X(t), V(t)), t \geq 0$, under appropriate assumptions on $r(\cdot)$ and $l(\cdot)$. Under stationarity, we show in Section 3 how these two functions appear in the study of level crossings, recurrence times, and points of reversal. In Section 4 we show how exact formulae for exit distributions, mean exit times, and other additive functionals are obtained from the appropriate differential equations. In Section 5 we investigate what happens if we increase the erraticism of our process by replacing $r(\cdot)$ and $l(\cdot)$ by $r(\cdot)+\lambda$ and $l(\cdot)+\lambda$, respectively, and letting $\lambda \rightarrow \infty$ : a link emerges with one-dimensional diffusions whose drift and diffusion coefficients involve the entities $r(x)-l(x)$ and $\gamma$. We conclude with a comment on the case of more than two possible values for the velocity.

\section{The model}

The stochastic process we consider here has two components, $X(t)$ and $V(t)$, where $X(t)$ denotes the position and $V(t)$ denotes the velocity at time $t(t \geq 0)$ of a particle moving on the real line $\mathbb{R}$. The velocity is assumed to take only two possible values, $\gamma$ or $-\gamma$ (where $\gamma>0$ ), and will be seen to jump from one value to the other only a finite number of times in any finite time interval, so that $V(t), t \geq 0$, is a (non-Markovian) pure-jump process. The particle is 
thus assumed to move with constant velocity between the consecutive random times at which it reverses its velocity from $\gamma$ to $-\gamma$ or vice versa. We will take $X(\cdot)$ to be continuous and $V(\cdot)$ to be right continuous. The occurrence of these reversals is specified informally (for the moment) as in the introduction above: if at time $t$ the particle is at position $x$ and moving with velocity $\gamma$, then its conditional probability of reversing its velocity before time $t+\Delta t$ is $r(x) \Delta t+o(\Delta t)$ as $\Delta t \rightarrow 0$, where $r(\cdot)$ is a nonnegative continuous function satisfying certain conditions to be stated below. Likewise, if its velocity is $-\gamma$, the conditional probability of a reversal before time $t+\Delta t$ is $l(x) \Delta t+o(\Delta t)$ as $\Delta t \rightarrow 0$, where $l(\cdot)$ is another nonnegative continuous function. To be more precise, given $X(t)=x$ and $V(t)=\gamma$, regardless of past history, the first reversal after time $t$ occurs at time $t+\tau$, where $\tau$ is random and has a conditional distribution with probability density function

$$
q(s)=r(x+\gamma s) \exp \left(-\int_{0}^{s} r(x+\gamma u) \mathrm{d} u\right), \quad s \geq 0,
$$

and with a possible atom $\exp \left(-\int_{0}^{\infty} r(x+\gamma u) \mathrm{d} u\right)$ at $\{\tau=\infty\}$. No such atom will arise below. The function $r(x+\gamma s), s \geq 0$, can be recognised as a 'failure rate' or 'hazard' function in the language of reliability theory and it is an elementary fact that $\exp \left(-\int_{0}^{s} r(x+\gamma u) \mathrm{d} u\right)$ is the conditional probability that $\tau \geq s$ (i.e. the so-called reliability function). Thus, during the time interval $[t, t+\tau]$, the motion of the particle is given by $X(t+s)=x+\gamma s$ and immediately after time $t+\tau$ we have $X\left(t+\tau+s^{\prime}\right)=x+\gamma \tau-\gamma s^{\prime}$ until the next reversal.

If we are given an initial position and an initial velocity, then the above specification defines a stochastic process $(X(t), V(t)), t \geq 0$, which is Markovian (in fact, strong Markov, with stationary transition probabilities), although in general neither the marginal process $X(t)$ nor the marginal process $V(t)$ is Markovian.

On the space of bounded Borel functions $f(x, i), x \in \mathbb{R}, i= \pm \gamma$, we define the operators

$$
\left(T_{t} f\right)(x, i)=\mathrm{E}(f(X(t), V(t)) \mid X(0)=x, V(0)=i), \quad t \geq 0,
$$

which form the contraction semigroup of the Markov process $(X(t), V(t)), t \geq 0$. Adopting the notation of [4], whereby the function $f(x, i), x \in \mathbb{R}, i= \pm \gamma$, is denoted by the vector $\left(f_{\gamma}(x), f_{-\gamma}(x)\right)^{\top}$ with $f_{i}(x)=f(x, i)$, we easily obtain as in [4] the infinitesimal generator $G$ of the semigroup $T_{t}, t \geq 0$, defined by

$$
G f=\lim _{\varepsilon \searrow 0} \frac{1}{\varepsilon}\left(T_{\varepsilon} f-f\right)
$$

for functions $f$ with a continuous first derivative $f^{\prime}$ with respect to $x$ (in which case the convergence in (2.1) is uniform on any bounded $x$-interval). In fact, noting that, by the continuity of the functions $r(\cdot), l(\cdot)$, and $f(\cdot, i)$,

$$
\left(T_{\varepsilon} f\right)(x, \gamma)=(1-r(x) \varepsilon) f(x+\gamma \varepsilon, \gamma)+r(x) \varepsilon f(x,-\gamma)+o(\varepsilon),
$$

we see that $G$ is given by the (variable) matrix

$$
G=\left(\begin{array}{cc}
\gamma \frac{\mathrm{d}}{\mathrm{d} x}-r(\cdot) & r(\cdot) \\
l(\cdot) & -\gamma \frac{\mathrm{d}}{\mathrm{d} x}-l(\cdot)
\end{array}\right),
$$


i.e.

$$
G\left(\begin{array}{c}
f_{\gamma} \\
f_{-\gamma}
\end{array}\right)=\left(\begin{array}{c}
\gamma f_{\gamma}^{\prime}-r\left(f_{\gamma}-f_{-\gamma}\right) \\
-\gamma f_{-\gamma}^{\prime}+l\left(f_{\gamma}-f_{-\gamma}\right)
\end{array}\right)
$$

where we have omitted the variable $x$ in the notation. Compare with [4], where of course $r(\cdot)$ and $l(\cdot)$ are both equal to a constant $\lambda$.

Under appropriate conditions on $r(\cdot)$ and $l(\cdot)$, we can obtain a stationary distribution for the process $(X(t), V(t)), t \geq 0$, something which does not exist if $r(\cdot)$ and $l(\cdot)$ are constant. The forward (adjoint) differential equation for a stationary probability density function $\theta(x, i)$, $x \in \mathbb{R}, i= \pm \gamma$, is

$$
\left(\theta_{\gamma}, \theta_{-\gamma}\right) G^{*}=(0,0)
$$

where, as before, $\theta_{i}(x)=\theta(x, i)$ and

$$
G^{*}=\left(\begin{array}{cc}
-\gamma \frac{\mathrm{d}}{\mathrm{d} x}-r(.) & r(\cdot) \\
l(\cdot) & \gamma \frac{\mathrm{d}}{\mathrm{d} x}-l(\cdot)
\end{array}\right) .
$$

(Apply an integration by parts to the condition that $\int_{-\infty}^{\infty} \sum_{i= \pm \gamma} \theta_{i}(x)(G f)_{i}(x) \mathrm{d} x=0$ for sufficiently many $f$.)

The unique solution of (2.2), i.e. of the system

$$
\gamma \theta_{i}^{\prime}(x)=-r(x) \theta_{\gamma}(x)+l(x) \theta_{-\gamma}(x), \quad i= \pm \gamma,
$$

which is a probability density function, is given by

$$
\theta_{\gamma}(x)=\theta_{-\gamma}(x)=C_{a} \exp \left(-\int_{a}^{x} \frac{r(u)-l(u)}{\gamma} \mathrm{d} u\right),
$$

where $a$ is an arbitrary fixed number and $C_{a}$ is a normalising constant, provided the exponential in (2.3) is integrable. This is certainly the case if the following condition holds, which we will assume from now on.

Assumption 2.1. There exist constants $k>0$ and $m>0$ such that $r(u)-l(u) \geq k$ for $u \geq m$ and $l(u)-r(u) \geq k$ for $u \leq-m$.

For convenience, we will use the notation

$$
h_{a}(x)=\exp \left(-\int_{a}^{x} \frac{r(u)-l(u)}{\gamma} \mathrm{d} u\right),
$$

and we will write $\theta(x)$ for $\theta_{\gamma}(x)=\theta_{-\gamma}(x)$, so that from now on $\theta(x)$ stands for $\theta(x, i)$. Trivially, (2.3) implies that $C_{a}=\theta(a)$, so that

$$
\theta(x)=\theta(a) h_{a}(x) .
$$

Proposition 2.1. Under Assumption 2.1, there exists a unique stationary distribution with density given by (2.3).

Note that results from the classical theory of Markov processes imply that

$$
\lim _{t \rightarrow \infty} \mathrm{P}(a<X(t)<b, V(t)=j \mid X(0)=x, V(0)=i)=\int_{a}^{b} \theta(y) \mathrm{d} y
$$

for any interval $(a, b)$, bounded or not, and any $x, i, j$. We will make use of this below. 
At this point it is worth giving a simple example of a 'random pendulum'. Let $\gamma=1$. Take $r(x)$ to be equal to $x$ for $x \geq 0$ and equal to 0 for $x<0$. Likewise, take $l(x)$ to be $-x$ if $x \leq 0$ and 0 if $x>0$. Note that $r(x), x \geq 0$, is the hazard function of the Weibull (Rayleigh) distribution with probability density function $x \exp \left(-\frac{1}{2} x^{2}\right), x \geq 0$. In this case the density of the stationary distribution is just the standard normal density. The motion is a pure oscillation in the sense that, if the particle starts from 0 with velocity 1 , it continues its upward motion until the time of first reversal (which has the above Rayleigh distribution), then switches to a downward motion which cannot be interrupted as long as the particle is on the positive half-line. After the first downcrossing of level 0 , the hazard function $l(\cdot)$ comes into play, representing the particle's propensity to turn upwards, and the motion undergoes a reversal at some negative level which has as distribution the mirror image of the above Rayleigh distribution. The particle then proceeds uninterrupted to level 0 , etc. Such an oscillation can easily be studied through its renewal structure. Below we will look at a simple modification of this example.

\section{Level crossings, recurrence times, and reversals}

In the presence of a stationary distribution simple stationarity and/or renewal arguments can give us a handful of expected values without recourse to the differential equations set up in Section 4 for the calculation of other related entities. This hinges on the following proposition, in which 'stationarity' means that the process $(X(t), V(t)), t \geq 0$, is started in its stationary distribution.

Proposition 3.1. Under stationarity, for each $x \in \mathbb{R}$, the expected number of crossings of level $x$ in a unit of time is $2 \gamma \theta(x)$, where $\theta$ is the density of the stationary distribution given by (2.3).

This is a discrete mean local time at level $x$.

Proof of Proposition 3.1. Consider a range $[a, b]$ of values $x$ and, for $t \in[0,1]$, define $Y(t)=X(t)$ if $a \leq X(t) \leq b, Y(t)=a$ if $X(t)<a$, and $Y(t)=b$ if $X(t)>b$. The total variation $U$ of $Y(t), 0 \leq t \leq 1$, is $U=\int_{0}^{1}\left|Y^{\prime}(t)\right| \mathrm{d} t=\gamma \mu\{t \in[0,1]: a \leq X(t) \leq b\}$ (where $\mu$ denotes the Lebesgue measure), since $Y^{\prime}(t)= \pm \gamma$ almost everywhere on $\{t \in[0,1]: a \leq$ $X(t) \leq b\}$. For each $x \in[a, b]$, let $N(x)$ be the number of values of $t \in[0,1]$ for which $X(t)=x$. The function $N(\cdot)$ is the Banach indicatrix of $X(\cdot)$ and, by Banach's well-known theorem $U=\int_{a}^{b} N(x) \mathrm{d} x$; hence,

$$
\begin{aligned}
\int_{a}^{b} \mathrm{E} N(x) \mathrm{d} x & =\mathrm{E}\left(\int_{a}^{b} N(x) \mathrm{d} x\right) \\
& =\mathrm{E} U \\
& =\gamma \mathrm{E} \mu\{t \in[0,1]: a \leq X(t) \leq b\} \\
& =\gamma \mathrm{E} \int_{0}^{1} \mathbf{1}_{[a, b]}(X(t)) \mathrm{d} t \\
& =\gamma \int_{0}^{1} \mathrm{E} \mathbf{1}_{[a, b]}(X(t)) \mathrm{d} t \\
& =\gamma \mathrm{E} \mathbf{1}_{[a, b]}(X(0)) \\
& =\gamma \int_{a}^{b} 2 \theta(x) \mathrm{d} x .
\end{aligned}
$$


Since this is true for arbitrary $[a, b]$, we deduce that, for almost all $x, \mathrm{E} N(x)=2 \gamma \theta(x)$, and it is easy to show that $\mathrm{E} N(x)$ is a continuous function of $x$.

Corollary 3.1. Under stationarity, for each $x \in \mathbb{R}$, the expected number of upcrossings of level $x$ in a unit of time is $\gamma \theta(x)$ and if $\tau=\min \{t>0: X(t)=x, V(t)=\gamma\}$ then $\mathrm{E}(\tau \mid X(0)=x$, $V(0)=\gamma\}=\gamma^{-1} \theta(x)^{-1}$.

In fact, since upcrossings and downcrossings alternate and the process is stationary, the expected number of upcrossings must be equal to the expected number of downcrossings. The second assertion follows from standard renewal theory: since the process $(X(t), V(t)), t \geq 0$, has the strong Markov property, the times of consecutive upcrossings of level $x$ form a stationary renewal process.

We can use the renewal approach to obtain the mean time between an upcrossing and the subsequent downcrossing of the same level.

Corollary 3.2. If $x \in \mathbb{R}$ and $\sigma=\min \{t>0: X(t)=x, V(t)=-\gamma\}$, then

$$
\mathrm{E}(\sigma \mid X(0)=x, V(0)=\gamma)=\frac{2}{\gamma} \int_{x}^{\infty} \exp \left(-\int_{x}^{y} \frac{r(u)-l(u)}{\gamma} \mathrm{d} u\right) \mathrm{d} y .
$$

Proof. Assume that $X(0)=x$ and $V(0)=\gamma$, and denote by $\mathrm{P}^{\prime}$ and $\mathrm{E}^{\prime}$ the conditional probabilities and expectations given $X(0)=x$ and $V(0)=\gamma$. Let $\tau_{0}=0, \tau_{1}, \tau_{2}, \ldots$ be the consecutive upcrossings of level $x$, and let $\sigma_{0}, \sigma_{1}, \sigma_{2}, \ldots$ be the consecutive downcrossings, so that $0<\sigma_{0}<\tau_{1}<\sigma_{1}<\tau_{2}<\cdots$. The theory of renewal processes with two components implies that

$$
\lim _{t \rightarrow \infty} \mathrm{P}^{\prime}(X(t) \geq x)=\lim _{t \rightarrow \infty} \mathrm{P}^{\prime}\left(t \in \bigcup_{k=0}^{\infty}\left(\tau_{k}, \sigma_{k}\right)\right)=\frac{\mathrm{E}^{\prime} \sigma_{0}}{\mathrm{E}^{\prime} \tau_{1}} .
$$

Comparing with (2.6) we see that

$$
\frac{\mathrm{E}^{\prime} \sigma_{0}}{\mathrm{E}^{\prime} \tau_{1}}=\int_{x}^{\infty} 2 \theta(y) \mathrm{d} y .
$$

By Corollary 3.1 and in view of (2.5),

$$
\mathrm{E}^{\prime} \sigma_{0}=\gamma^{-1} \theta(x)^{-1} \int_{x}^{\infty} 2 \theta(y) \mathrm{d} y=\frac{2}{\gamma} \int_{x}^{\infty} h_{x}(y) \mathrm{d} y .
$$

This proves (3.1) by (2.4).

Another simple fact worth mentioning is that if $[a, b]$ is any bounded interval then the expected number of points $(t, x) \in(0,1] \times[a, b]$ at which the process $(X(t), V(t)))$ undergoes a reversal from 'up' to 'down' (in the sense that $X(t)=x, V(t-)=\gamma$, and $V(t+)=-\gamma$ ) is, under stationarity, $\int_{a}^{b} \theta(x) r(x) \mathrm{d} x$. This can easily be proved by considering nested partitions of $(0,1]$.

\section{Exit distributions, mean exit times, and additive functionals}

Suppose that $-\infty<a<b<\infty$ and that $a<X(0)<b$. Define $T=\min \{t>0$ : $X(t) \in\{a, b\}\}$, and let $B$ be the event $\{X(T)=b\}$. Since the process $(X(t), V(t)), t \geq 0$, has a stationary distribution (and, hence, is 'positive recurrent'), all conditional expectations $\mathrm{E}(T \mid X(0)=x, V(0)=i), x \in(a, b), i= \pm \gamma$, are finite. 
For $x \in(a, b)$, consider the probabilities

$$
\phi(x, i)=\phi_{i}(x)=\mathrm{P}(B \mid X(0)=x, V(0)=i), \quad i= \pm \gamma,
$$

and, for a fixed continuous (in $y)$ function $g(y, i)(a \leq y \leq b, i= \pm \gamma)$, the expectations

$$
\psi(x, i)=\psi_{i}(x)=\mathrm{E}\left(\int_{0}^{T} g(X(s), V(s)) \mathrm{d} s \mid X(0)=x, V(0)=i\right), \quad i= \pm \gamma .
$$

In line with diffusion theory and the theory of the two-state random evolution, the functions $\phi_{i}$ and $\psi_{i}$ can be shown to satisfy $G \phi=\mathbf{0}$ (where $\mathbf{0}=(0,0)^{\top}$ ) and $G \psi=-g$, respectively, i.e. the system

$$
\begin{aligned}
\gamma \phi_{\gamma}^{\prime}(x) & =r(x)\left(\phi_{\gamma}(x)-\phi_{-\gamma}(x)\right), \\
\gamma \phi_{-\gamma}^{\prime}(x) & =l(x)\left(\phi_{\gamma}(x)-\phi_{-\gamma}(x)\right),
\end{aligned}
$$

and the system

$$
\begin{aligned}
\gamma \psi_{\gamma}^{\prime}(x) & =r(x)\left(\psi_{\gamma}(x)-\psi_{-\gamma}(x)\right)-g_{\gamma}(x), \\
\gamma \psi_{-\gamma}^{\prime}(x) & =l(x)\left(\psi_{\gamma}(x)-\psi_{-\gamma}(x)\right)+g_{-\gamma}(x),
\end{aligned}
$$

respectively. The relevant boundary conditions are $\phi_{\gamma}(b-)=1, \phi_{-\gamma}(a+)=0, \psi_{\gamma}(b-)=0$, and $\psi_{-\gamma}(a+)=0$.

These differential equations can be derived either from first principles differentiation or, as the referee has suggested, from the standard martingale approach to such problems. See [3] for the case of diffusion. As a matter of fact, if, for example, $\zeta(x, i)$ is a solution of $G \zeta=-g$, we can appeal directly to Dynkin's formula in the form

$$
\begin{aligned}
& \mathrm{E}(\zeta(X(T), V(T)) \mid X(0)=x, V(0)=i) \\
& \quad=\zeta(x, i)+\mathrm{E}\left(\int_{0}^{T}(G \zeta)(X(s), V(s)) \mathrm{d} s \mid X(0)=x, V(0)=i\right),
\end{aligned}
$$

which is valid since $\mathrm{E}(T \mid X(0)=x, V(0)=i)<\infty$. See [1, p. 133]. This implies that, subject to the right boundary conditions, $\zeta(x, i)=\psi(x, i)$. For the alternative approach via differentiation, note, for example, that, for small $h>0$,

$$
\begin{aligned}
\phi(x, \gamma) & =\mathrm{E}(\phi(X(h), V(h)) \mid X(0)=x, V(0)=\gamma) \\
& =\phi(x+\gamma h, \gamma)(1-r(x) h)+\phi(x+O(h),-\gamma) r(x) h+o(h),
\end{aligned}
$$

etc.

The solution of the system (4.2), (4.3) is elementary. Subtracting (4.3) from (4.2) we obtain an equation which we can readily solve for $\phi_{\gamma}(x)-\phi_{-\gamma}(x)$. Adding (4.3) to (4.2) we obtain another equation which gives us $\phi_{\gamma}(x)+\phi_{-\gamma}(x)$ in terms of $\phi_{\gamma}(x)-\phi_{-\gamma}(x)$. Taking account of the boundary conditions we arrive at the following exact solution.

Proposition 4.1. We have

$$
\phi_{ \pm \gamma}(x)=C\left[1+\int_{a}^{x} \frac{r(u)+l(u)}{\gamma} \exp \left(\int_{a}^{u} \frac{r(v)-l(v)}{\gamma} \mathrm{d} v\right) \mathrm{d} u \pm \exp \int_{a}^{x} \frac{r(u)-l(u)}{\gamma} \mathrm{d} u\right],
$$

where $C$ is such that $\phi_{\gamma}(b-)=1$. 
The solution of the system (4.4), (4.5) is no less elementary. The only difference is that the equation we obtain by subtracting (4.5) from (4.4) requires an integrating factor for its solution. Explicit expressions are thus obtained for the expectations $\psi_{i}(x)$ in (4.1); however, these are too lengthy to deserve displaying here. Discontinuous additive functionals, such as the number of 'reversals' of the process in a time interval $\left(t_{1}, t_{2}\right)$, can be handled similarly.

The formulae become less daunting in the special case $g(x, i) \equiv 1$. In this case the functions $\psi_{i}(x), a<x<b$, in (4.1) are the mean exit times from the interval $(a, b)$, given $X(0)=x$ and $V(0)=i$. In this special case (4.4) and (4.5) yield the following result.

Proposition 4.2. The mean exit times $\psi_{i}(x)=\mathrm{E}(T \mid X(0)=x, V(0)=i), x \in(a, b)$, $i= \pm \gamma$, can be obtained from

$$
\psi_{\gamma}(x)-\psi_{-\gamma}(x)=h_{a}(x)^{-1}\left(\psi_{\gamma}(a+)-2 \gamma^{-1} \int_{a}^{x} h_{a}(u) \mathrm{d} u\right)
$$

and

$$
\begin{aligned}
\psi_{\gamma}(x)+\psi_{-\gamma}(x)= & \psi_{\gamma}(a+) \\
& +\gamma^{-1} \int_{a}^{x}(r(u)+l(u)) h_{a}(u)^{-1}\left(\psi_{\gamma}(a+)-2 \gamma^{-1} \int_{a}^{u} h_{a}(v) \mathrm{d} v\right) \mathrm{d} u,
\end{aligned}
$$

where $h_{a}(x)$ is given by (2.4) and the constant $\psi_{\gamma}(a+)$ is determined by virtue of the boundary condition $\psi_{\gamma}(b-)=0$.

Having exact expressions for exit distributions and mean exit times may be a modest enough result, but it is worth remembering that the corresponding entities for one-dimensional diffusions determine the scale measure and the speed measure which are defining characteristics of such diffusions. In this connection the asymptotic results of the next section may be of some interest.

\section{Some asymptotics}

Let us increase the erraticism of our process as follows. Replace $r(x)$ and $l(x)$ by $r^{*}(x)=$ $r(x)+\lambda$ and $l^{*}(x)=l(x)+\lambda$, respectively, where $\lambda$ is a positive number, and denote by $\phi_{i}^{*}(x), i= \pm \gamma$, the corresponding probabilities of exit through $b$ and by $\psi_{i}^{*}(x), i= \pm \gamma$, the corresponding mean exit times from $(a, b)$. We intend to let $\lambda \rightarrow \infty$. A large value of $\lambda$ means of course that the process undergoes rapid reversals. Routine calculations show that

$$
\lim _{\lambda \rightarrow \infty} \phi_{i}^{*}(x)=\frac{S(x)}{S(b)}, \quad i= \pm \gamma
$$

where

$$
S(x)=\int_{a}^{x} \exp \left(\int_{a}^{u} \frac{r(v)-l(v)}{\gamma} \mathrm{d} v\right) \mathrm{d} u .
$$

Thus, we have the following result.

Proposition 5.1. As $\lambda \rightarrow \infty$, the exit probabilities $\phi_{i}^{*}(x)$ converge to those of any onedimensional diffusion process with drift $\mu(x)$ and diffusion coefficient $\sigma^{2}(x)$ such that

$$
\frac{\mu(x)}{\sigma^{2}(x)}=-\frac{r(x)-l(x)}{2 \gamma} .
$$


The function $S(x)$ given in (5.1) is the scale function of such a diffusion (see [2]). We see that, as $\lambda \rightarrow \infty$, the effect of $V(0)=i$ wears off asymptotically due to the rapid reversals of direction.

For the mean exit times $\psi_{i}^{*}(x)$, we can show that

$$
\begin{aligned}
\lim _{\lambda \rightarrow \infty} \frac{\psi_{\gamma}^{*}(x)}{\lambda}= & \lim _{\lambda \rightarrow \infty} \frac{\psi_{-\gamma}^{*}(x)}{\lambda} \\
= & 2 \gamma^{-2}\left(\int_{a}^{x} h_{a}(u)^{-1} \mathrm{~d} u\right)\left(\int_{a}^{b} h_{a}(u)^{-1} \mathrm{~d} u\right)^{-1} \int_{a}^{b} h_{a}(u)^{-1} \int_{a}^{u} h_{a}(v) \mathrm{d} v \mathrm{~d} u \\
& -2 \gamma^{-2} \int_{a}^{x} h_{a}(u)^{-1} \int_{a}^{u} h_{a}(v) \mathrm{d} v \mathrm{~d} u .
\end{aligned}
$$

A glance at the top of page 197 of [2] confirms the truth of the following.

Proposition 5.2. When $\lambda \rightarrow \infty$, the functions $\psi_{i}^{*}(x) / \lambda, a<x<b$, both converge to the mean exit times of a one-dimensional diffusion with drift $\mu(x)=-\gamma(r(x)-l(x)) / 2$ and diffusion coefficient $\sigma^{2}(x)=\gamma^{2}$.

The speed density of this diffusion corresponding to the scale density $h_{a}(x)^{-1}$ appearing in (5.1) is the function $\gamma^{-2} h_{a}(x)$.

Let us specialise these asymptotics to the random pendulum described at the end of Section 2 , by setting $r^{*}(x)=\lambda+\max \{x, 0\}$ and $l^{*}(x)=\lambda+\max \{-x, 0\}(\lambda>0)$. In this case it is easy to prove that

$$
\lim _{\lambda \rightarrow \infty} \phi_{i}^{*}(x)=\frac{\int_{a}^{x} \exp \left(u^{2} / 2 \gamma\right) \mathrm{d} u}{\int_{a}^{b} \exp \left(u^{2} / 2 \gamma\right) \mathrm{d} u}, \quad i= \pm \gamma .
$$

The expression on the right-hand side can be recognised as the probability that an OrnsteinUhlenbeck process with drift $-\gamma u / 2$ at $u$ and diffusion coefficient $\gamma^{2}$, starting from $x$, will hit $b$ before $a$. Likewise, the limit (5.2) in this special case can be recognised as the mean exit time from $(a, b)$ of this same Ornstein-Uhlenbeck process.

According to Proposition 5.2, mean exit times from $(a, b)$ tend to $\infty$ when $\lambda \rightarrow \infty$, for every interior point $x$. It is natural to see what happens if we speed up our process by a factor of $\lambda$. For any random oscillation $(X(t), V(t)), t \geq 0$, the component $V(t)$ is the derivative of $X(t)$ (except at reversal points). We can change the time scale by a factor $\beta>0$ by defining $\left(X_{\beta}(t), V_{\beta}(t)\right)=(X(\beta t), \beta V(\beta t)), t \geq 0$. This new process is itself a random oscillation with parameters $\bar{r}(\cdot)=\beta r(\cdot), \bar{l}(\cdot)=\beta l(\cdot)$, and $\bar{\gamma}=\beta \gamma$. The functions $h_{a}(\cdot)$ are the same for $X(\cdot)$ and $X_{\beta}(\cdot)$. Returning to the process with propensity functions $r(\cdot)+\lambda$ and $l(\cdot)+\lambda$, if we introduce the corresponding scaled process with $\beta=\lambda$, we see that its mean exit times $\bar{\psi}_{i}(x)$ are equal to $\psi_{i}^{*}(x) / \lambda(a<x<b)$ and, therefore, converge, when $\lambda \rightarrow \infty$, to those displayed on the right-hand side of (5.2). Combining this with Propositions 5.1 and 5.2 we have the following result.

Theorem 5.1. The exit distributions and mean exit times of the random oscillation with parameters $\tilde{r}(\cdot)=\lambda(r(\cdot)+\lambda), \tilde{l}(\cdot)=\lambda(l(\cdot)+\lambda)$, and $\tilde{\gamma}=\lambda \gamma$ converge, as $\lambda \rightarrow \infty$, for both $V(0)= \pm \gamma$, to the exit distributions and mean exit times of the diffusion with drift $\mu(x)=-\gamma(r(x)-l(x)) / 2$ and diffusion coefficient $\sigma^{2}(x)=\gamma^{2}$.

A different example with growing erraticism is the following. Take $\gamma=1$ and, for $x \geq 0$, let $r(x)=(L+1) x$ and $l(x)=L x$, while, for $x<0$, let $r(x)=-L x$ and $l(x)=-(L+1) x$, where $L>0$. The diffusion which appears as above in the asymptotics of this random oscillation 
when $L \rightarrow \infty$ is the one with (for $x>0)$ drift $\mu(x)=-\frac{1}{2}\left(1+1 / x^{2}\right)$ and diffusion coefficient $\sigma^{2}(x)=1 / x$.

\section{Comment on the multispeed case}

The differential equations corresponding to a random oscillation in which the two possible values of the velocity are $\gamma_{1}$ and $\gamma_{2}$, where $\gamma_{2}<0<\gamma_{1}$, but $-\gamma_{2} \neq \gamma_{1}$, can be solved just as easily as in the case $-\gamma_{2}=\gamma_{1}$. This is not so for the obvious extension of the model to the case of more than two possible values, say $\gamma_{1}, \gamma_{2}, \ldots, \gamma_{N}$, although the corresponding differential equations can be set up very easily. The formal structure of such an extension can be defined as follows. Given $X(t)=x$ and $V(t)=\gamma_{i}$, the first change of velocity after time $t$ occurs at time $t+\tau$ and is a change to velocity $\gamma_{\nu}$, where $\tau$ and $v$ are random with conditional joint distribution given by

$$
\mathrm{P}\left(\tau \in \mathrm{d} s, v=j \mid X(t)=x, V(t)=\gamma_{i}\right)=r_{i j}\left(x+\gamma_{i} s\right) \exp \left(-\int_{0}^{s} \sum_{k \neq i} r_{i k}\left(x+\gamma_{i} u\right) \mathrm{d} u\right) \mathrm{d} s
$$

for $s>0$ and $j \neq i$. Here appropriate assumptions have to be made for the propensity functions $r_{i j}(x)$.

\section{Acknowledgement}

Thanks are due to the anonymous referee for several helpful comments and for curbing the author's penchant for a rather leisurely style of presentation in the original manuscript.

\section{References}

[1] Dynkin, E. B. (1965). Markov Processes, Vol. I. Springer, Berlin.

[2] Karlin, S. and Taylor, H. M. (1981). A Second Course in Stochastic Processes. Academic Press, New York.

[3] Klebaner, F. C. (1998). Introduction to Stochastic Calculus with Applications. Imperial College Press, London.

[4] PInsky, M. A. (1991). Lectures on Random Evolution. World Scientific, River Edge, NJ. 